\title{
On Ray's theorem for weak firmly nonexpansive mappings in Hilbert Spaces
}

\author{
Tamer Nabil ${ }^{1}$ and Ahmed H. Soliman ${ }^{2}$ \\ ${ }^{1}$ Suez Canal University, Faculty of Computers and Informatics, Department of Basic Science, Ismailia, Egypt \\ 1t_3bdelsadek@yahoo.com \\ ${ }^{2}$ Department of Mathematics, Faculty of Science, Al-Azhar University, Assiut 71524, Egypt \\ 2ahsolimanm@gmail.com
}

ABSTRACT

In this work, we introduce notions of generalized firmly nonexpansive (G-firmly nonexpansive) and fundamentally firmly nonexpansive (F-firmly nonexpansive) mappings and utilize to the same to prove Ray's theorem for G-firmly and F-firmly nonexpansive mappings in Hilbert Spaces. Our results extend the result due to F. Kohsaka [ Ray's theorem revisited: a fixed point free firmly nonexpansive mapping in Hilbert spaces, Journal of Inequalities and Applications (2015) 2015:86 ].

Keywords. Ray's theorem; generalized firmly nonexpansive mapping; fundamentally firmly nonexpansive mapping; fixed point; Hilbert space.

\section{Mathematics Subject Classification: 47H10, 54H10, 54H25.}

\section{INTRODUCTION and PRELIMINARIES}

Let $H$ be a real Hilbert space. The inner product and the induced norm on $H$ are denoted by $\langle.,$.$\rangle and \|$.$\| respectively.$

The dual space of a Banach space $X$ is denoted $X^{*}$. Consider $K$ is nonempty closed convex subset of $H$. A mapping $T: K \rightarrow K$ is said to be nonexpansive mapping if

$$
\|T x-T y\| \leq\|x-y\|
$$

for all $x, y \in K$.

In 1965, Browder [1] showed that if $K$ is bounded, then every nonexpansive mapping on $K$ has a fixed point. In 1980, Ray [2] showed that the converse of Browder's theorem is true, i.e. every nonexpansive self mapping on $K$ has a fixed point, then $K$ is bounded. There are many versions of Ray's theorem for nonexpansive mapping. For examples, in 1987, Sine [3], proved Ray's theorem by applying a version of the uniform boundedness principle (see, for instance, [6]) and the convex combination of a sequence of a metric projections onto closed and convex sets. In 2010. Aoyama et al. [4], obtained a strong version of Ray's theorem for the class of $\lambda$-hybrid mappings in Hilbert spaces.

Recently, Kohsaka [5] given another proof of a strong version of Ray's theorem [4] ensuring that every unbounded closed convex subset of a Hilbert space admits a fixed point free firmly nonexpansive mapping. He used in his proof a version of uniform boundedness principle and single metric projection onto a closed and convex set.

In this paper, we define two new class of weaker firmly nonexpansive called G-firmly and F-firmly nonexpansive. We present new two versions of Ray's theorem for mappings satisfying the conditions of weaker firmly nonexpansive.

We begin with some notations and preliminaries.

Definition 1.1. [5] A mapping $T: K \rightarrow K$ is said to be firmly nonexpansive if

$$
\|T x-T y\|^{2} \leq\langle T x-T y, x-y\rangle
$$

for all $x, y \in K$.

Definition 1.2. [7] A linear subspace $M$ of a normed space $X$ is called proximinal (resp. Chebyshev) if for each $x \in X$, the set of best approximations to $x$ from $M$,

$$
P_{M}:=\left\{y \in M:\|x-y\|=\inf _{m \in M}\|x-m\|\right\},
$$

is nonempty (resp. a singleton). It well know that for each element of the Hilbert space there exist Chebyshev convex subset. 
Definition 1.3. [5] The mapping $P_{K}: H \rightarrow K$ which is defined by $P_{K} x=z_{x}$ for $x \in H$ such that $\left\|P_{K} x-x\right\| \leq\|y-x\|$ for all $y \in K$ is called the metric projection of $H$ onto $K$, therefore, $z=P_{K} x$ if and only if $\sup _{y \in K}\langle y-z, x-z\rangle \leq 0$ for all $(x, y) \in H \times K$.

Theorem 1.1. (A strong version of Rays theorem [4]) Let $K$ be a nonempty closed convex subset of a Hilbert space $H$. If every firmly nonexpansive self-mapping on $K$ has a fixed point, then $K$ is bounded.

\section{MAIN RESULTS}

We now present our new conditions of weak nonexpansive.

Definition 2.1. A self mapping $T$ on $K$ is said to be G-firmly nonexpansive if

$$
\frac{1}{3}\|x-T x\|^{2} \leq\langle T x-T y, x-y\rangle \Rightarrow\|T x-T y\|^{2} \leq\langle T x-T y, x-y\rangle, \forall x, y \in K .
$$

Proposition 2.1. Every firmly nonexpansive is G-firmly nonexpansive.

Remark 2.1. The converse of proposition 2.1 is not true as we will see in the following example.

Example 2.1. Define a mapping $T$ on $[0,4]$ such that $T x=0$ as $x \neq 4$ and $T x=0.5$ as $x=4$. Then $T$ is G-firmly nonexpansive but $T$ is not firmly nonexpansive. Where the inner product $\langle x, y\rangle=x$. $y$ for all real numbers $x$ and $y$.

Proof. It is clear that $T$ is not continuous, therefore it is not firmly nonexpansive. If $x<y$ and $x \in[0,2] \cup\{4\}$ and $y \in[0,4)$, then $\|T x-T y\|^{2} \leq\langle T x-T y, x-y\rangle$ holds. If $x \in(2,4)$ and $y=4$, then

$$
\frac{1}{3}\|x-T x\|^{2}=\frac{x^{2}}{3}>1,\langle T x-T y, x-y\rangle<1 \text { and } \frac{1}{3}\|y-T y\|^{2}>1 .
$$

Thus $T$ is generalized firmly nonexpansive mapping.-

Definition 2.2. A self mapping $T$ on $K$ is said to be F-firmly nonexpansive if

$$
\left\|T^{2} x-T y\right\|^{2} \leq\left\langle T^{2} x-T y, T x-y\right\rangle, \forall x, y \in K .
$$

Proposition 2.2. Every firmly nonexpansive is F-firmly nonexpansive.

Remark 2.2. The converse of proposition 2.2 is not true as we will see in the following example.

Example 2.2. Define the mapping $T$ on $[0,2]$ by

$$
T x=\left\{\begin{array}{lll}
0 & \text { if } & x \neq 2 \\
1 & \text { if } & x=2
\end{array}\right.
$$

And the inner product $\langle x, y\rangle=x$. $y$ for all real numbers $x$ and $y$.

Then $T$ is F-firmly nonexpansive but $T$ is not firmly nonexpansive.

Proof. Let $x=2$ and $y=1.5$. Then $\|T x-T y\|^{2}=1$, but $\langle T x-T y, x-y\rangle=0.5$. Thus $T$ is not firmly nonexpansive mapping.

If $x, y \in[0,2)$, then $\left\|T^{2} x-T y\right\|=0$ and $\left\langle T^{2} x-T y, T x-y\right\rangle=0$. If $x=2$ and $y \in[0,2)$, then we have that:

$$
\left\|T^{2} x-T y\right\|=1 \text { and }\left\langle T^{2} x-T y, T x-y\right\rangle=1 .
$$

Last case, if $x \in[0,2)$ and $y=2$, we get that: 


$$
\left\|T^{2} x-T y\right\|=1 \text { and }\left\langle T^{2} x-T y, T x-y\right\rangle=2 .
$$

Therefore $\mathrm{T}$ is F-firmly nonexpansive.

\section{Lemma 2.1.}

(1) the metric projection mapping $P_{K}$ (as in definition 1.3) of a Hilbert space $H$ onto a nonempty closed subset $K$ of $H$ is F-firmly nonexpansive,

(2) if $K$ be a nonempty closed convex subset of $H, a \in H$, and $T: K \rightarrow K$ such that $T x=P_{K}(x+a)$ for all $x \in K$. Then $T$ is a F-firmly nonexpansive self-mapping on $K$,

(3) $u \in K$ is fixed point of $T$ if and only if $\langle u, a\rangle=\sup \langle y, a\rangle$.

$$
y \in K
$$

Proof. (1) Let $x, y \in H$, thus we have that:

$$
\begin{aligned}
& \sup _{w \in K}\left\langle w-P_{K}^{2} x, P_{K} x-P_{K}^{2} x\right\rangle \leq 0 \text { and } \sup _{k \in K}\left\langle k-P_{K} y, y-P_{K} y\right\rangle \leq 0 \text { and hence } \\
& \left\|P_{K}^{2} x-P_{K} y\right\|^{2}-\left\langle P_{K}^{2} x-P_{K} y, P_{K} x-y\right\rangle= \\
& =\left\langle P_{K}^{2} x-P_{K} y, P_{K}^{2} x-P_{K} y\right\rangle-\left\langle P_{K}^{2} x-P_{K} y, P_{K} x-y\right\rangle \\
& =\left\langle P_{K}^{2} x-P_{K} y, P_{K}^{2} x-P_{K} y-P_{K} x+y\right\rangle \\
& =\left\langle P_{K}^{2} x-P_{K} y, y-P_{K} y\right\rangle+\left\langle P_{K}^{2} x-P_{K} y, P_{K}^{2} x-P_{K}^{2} x, P_{K} x-P_{K}^{2} x\right\rangle \\
& = \\
& =\sup _{w \in K}\left\langle w-P_{K} y, y-P_{K} y\right\rangle+\sup _{k \in K}\left\langle k-P_{K}^{2} x, P_{K} x-P_{K}^{2} x\right\rangle \\
& \leq 0 .
\end{aligned}
$$

Which implies that: $\left\|P_{K}^{2} x-P_{K} y\right\|^{2} \leq\left\langle P_{K}^{2} x-P_{K} y, P_{K} x-y\right\rangle$. Thus $P_{K}$ is F-firmly nonexpansive.

(2) $\|T x-T y\|^{2}=\left\|P_{K}(x+a)-P_{K}(y+a)\right\|^{2} \leq\left\langle P_{K}(x+a)-P_{K}(y+a), x+a-y-a\right\rangle=\langle T x-T y, x-y\rangle$.

Put, $x=T u$ and $v=y$, hence $T$ is a F-firmly nonexpansive self-mapping on $K$.

(3) $u \in F(T) \Leftrightarrow P_{K}(u+a)=u \Leftrightarrow \sup _{y \in K}\langle y-u, u+a-u\rangle \leq 0 \Leftrightarrow\langle u, a\rangle=\sup _{y \in K}\langle y, a\rangle$.

Lemma 2.2. The metric projection mapping of a Hilbert space $H$ onto a nonempty closed subset $K$ of $H$ is G-firmly nonexpansive. Furthermore, if $K$ be a nonempty closed convex subset of $H$, and $a \in H$, and $T: K \rightarrow K$ such that $T x=P_{K}(x+a)$ for all $x \in K$. Then $T$ is a G-firmly nonexpansive self-mapping on $K$ such that : $u \in K$ is fixed point of $T$ if and only if $\langle u, a\rangle=\sup _{y \in K}\langle y, a\rangle$.

Proof. Let $x, y \in K$, we have that:

$$
\|T x-T y\|^{2}=\left\|P_{K}(x+a)-P_{K}(y+a)\right\|^{2} \leq\left\langle P_{K}(x+a)-P_{K}(y+a), x+a-y-a\right\rangle=\langle T x-T y, x-y\rangle .
$$

Hence $T$ is a firmly self mapping on $K$. Then the same argument as in the proof of lemma 2.1 leads to $u \in F(T)$ if and only if $\langle u, a\rangle=\sup _{y \in K}\langle y, a\rangle$. 
We are now ready to introduce our new versions of Ray's theorem for weak firmly nonexpansive self-mappings.

Theorem 2.1 . ( F-firmly version of Ray's theorem ) Let $K$ be a nonempty closed convex of a Hilbert space

$H$. If the following fixed point property $(\mathrm{F})$ hold then $K$ is bounded.

(F) If every F-firmly nonexpansive mapping $T: K \rightarrow K$ has a fixed point.

Proof. Suppose that $K$ is unbounded. Thus there exist $x^{*} \in H$ such that $x^{*}(K)$ is unbounded (see, for instance, [6]). Then we have $a \in H$ such that : $\sup _{y \in K}\langle y, a\rangle=\infty$. Define $T x=P_{K}(x+a)$ and by (3) in Lemma 2.1, then $T$ is a fixed point free $\mathrm{F}$-firmly nonexpansive self mapping on $K$.

Theorem 2.2. ( G-firmly version of Ray's theorem) Let $K$ be a nonempty closed convex of a Hilbert space $H$. If the following fixed point property (E) hold then $K$ is bounded.

(E) If every G-firmly nonexpansive mapping $T: K \rightarrow K$ has a fixed point.

\section{ACKNOWLEDGMENTS}

The authors wish to thank the referees for the valuable remarks and commonts in the revision of this manuscript.

\section{REFERENCES}

[1] F. E. Browder, Non-expansive nonlinear operators in Banach spaces, Proc. Natl. Acad. Sci. USA 54 (1965) 1041-1044.

[2] W. O. Ray, The fixed point property and unbounded sets in Hilbert space, Trans. Am. Math. Soc. 258 (1980) 531- 537.

[3] R. Sine, On the converse of the nonexpansive map fixed point theorem for Hilbert space, Proc. Am. Math. Soc. 100 (1987) 489- 490.

[4] K. Aoyama, S. Lemoto, F. Kohsaka and W. Takahashi, Fixed point and ergodic theorems for $\lambda$-hybrid mapping in Hilber Spaces, J. Nonlinear Convex Anal. 11 (2010) 335-343.

[5] F. Kohsaka , Ray's theorem revisited: a fixed point free firmly nonexpansive mapping in Hilbert spaces, Journal of Inequalities and Applications (2015) 2015:86.

[6] H. Brezis, Functional Analysis, Sobolev Spaces and Partial Differential Equations. Springer, Berlin (2011).

[7] F. Deutsch, Linear selections for the metric projection, Journal of Functional Analysis 49 (1982), 269-292. 